\title{
Funding for spinal muscular atrophy research must continue
}

\author{
Melissa Bowerman*,1,2,3 (iD \\ ${ }^{1}$ School of Medicine, Keele University, Keele, ST5 5BG, UK \\ ${ }^{2}$ Institute for Science \& Technology in Medicine, Stoke-on-Trent, ST4 7QB, UK \\ ${ }^{3}$ Wolfson Centre for Inherited Neuromuscular Disease, RJAH Orthopaedic Hospital, Oswestry, SY10 7AG, UK \\ *Author for correspondence: m.bowerman@keele.ac.uk
}

First draft submitted: 8 February 2019; Accepted for publication: 22 February 2019; Published online: 4 June 2019

Keywords: fundamental research • funding • spinal muscular atrophy • smn • therapy $\bullet$ translational research

Spinal muscular atrophy (SMA) is a devastating neuromuscular disorder and is the leading genetic cause of infant mortality [1,2]. SMA is a monogenic disease caused by complete loss of the SMN1 gene and the full-length (FL) functional SMN protein it produces [3]. A recent duplication in the human genome, however, has resulted in a second almost identical copy of $S M N 1$, called $S M N 2$, which, due to a silent point mutation, mostly produces a truncated nonfunctional protein [4]. The SMN2 gene nevertheless retains the ability to generate a small amount of FL protein, thus allowing the survival of individuals born with homozygous deletions/mutations within SMN1 [4]. A total absence of SMN protein is indeed not compatible with life [5]. Furthermore, the number of SMN2 copies varies from person to person and there is a clear and accepted negative correlation between the number of SMN2 copies (i.e., FL SMN protein produced) and severity of disease manifestation [6].

The identification of SMN as the gene that causes SMA dates back to 1995 [3]. Since that seminal discovery, a series of fundamental, pre-clinical and clinical research endeavours have been aimed at identifying and developing pharmacological strategies that could increase SMN abundance, either by exogenously re-introducing the SMN1 gene or by promoting the production of FL SMN protein from the SMN2 gene [7]. This arduous and approximately 25-year journey, which was supported by the entire SMA community (fundamental researchers, clinicians, families, patients and funders), reached its intended goal in December 2016, when the first gene therapy for SMA was approved by the US FDA for all SMA patients. It was subsequently approved by the European Medicines Agency (EMA) in June 2017. The drug, an antisense oligonucleotide that promotes FL SMN expression from SMN2, is sold under the commercial name of Spinraza ${ }^{T M}$ by Ionis and Biogen [8].

Saying that the approval of Spinraza ${ }^{T M}$ has changed SMA research would be quite an understatement. It has in fact completely transformed both the SMA therapeutic landscape and the SMA patient population $[7,8]$. Indeed, in a blink of an eye, we went from having no available therapies to having one with the potential to completely alter the fate of SMA children. Newly diagnosed patients that typically could not expect to live beyond their second birthday, now not only live beyond that timeframe, but do so by reaching motor function milestones that surpass what was ever thought possible $[9,10]$. Our research and clinical priorities had to therefore naturally adapt and shift overnight [11]. Shift yes, but not stop. Spinraza is an incredible life-changing treatment but, unfortunately, not a cure [8]. Indeed, treated SMA patients can still display neuromuscular symptoms and decline, and for reasons still unknown, patients respond differently to the treatment, roughly dividing into groups of responders that improve, responders that plateau and non-responders [8,12-15]. While recent data support an increased benefit in patients treated pre-symptomatically, the 'earlier the better' dogma does not always hold true $[8,16]$. Furthermore, it remains unclear how Spinraza will be beneficial in older patients, in which significant neuromuscular decline has already occurred, and can most likely not be recovered $[8,17]$. Thus, more research into factors that influence the efficacy of Spinraza and its impact on different patient populations is of essence [8].

While the issues discussed above relate to Spinraza, similar inquiries and investigations will most likely arise following the approval of other SMN gene therapies (e.g. the Avexis/Novartis AAV9-SMN1 viral therapy and orally available small molecules by Roche and Novartis) that are anticipated to be approved by regulatory bodies

Future Medicine 
in the next few years, if not sooner [11]. Furthermore, once more than one $S M N$ gene therapy is available on the market, studies will need to be undertaken to better understand how and if these pharmacological compounds, which are administered via different delivery routes and have distinct modes of action, can be combined to optimize the benefits of increased SMN expression in all cells and tissues [7]. It will, therefore, be imperative that these investigations be performed, which to date have mostly been limited by access to these pharmacological compounds due to intellectual property (IP) and/or commercial-related issues. Hopefully, $S M N$ gene therapies will be made more readily available to both fundamental researchers and clinicians in the near future to ensure that relevant combinatorial studies can be undertaken.

Unfortunately, amid the positive enthusiasm generated by these life-changing $S M N$ gene therapies, there has been an underlying sentiment from the general public and research communities that further funding of SMA research is futile as the disease is now cured. We have indeed personally encountered such thoughts from specialist and nonspecialist grant and manuscript reviewers, and have heard similar feedback from several colleagues and community members. This reaction is understandable due to the success of Spinraza being almost miraculous in terms of its ability to prevent early death and significantly impact on quality of life of SMA patients and their families [8]. Nonetheless, even patients and their families are sometimes hesitant to undergo Spinraza treatment, whether it be due to the potential of unwanted side effects of either the drug or the lumbar puncture delivery method, its life-long high cost, the difficulties with insurance coverage and/or the uncertainty of long-term effectiveness $[8,18]$.

We thus find ourselves at a crucial junction and it is our duty, as researchers, citizens and members of the SMA community, to choose the best direction. New SMA research will inevitably have to take into account the SMN gene therapies that are either currently approved and available or that will be in the next few years. However, there are still large gaps in our knowledge that need to be filled to ensure that all SMA patients receive the best care and support throughout their entire life course $[7,8,11]$.

Indeed, we still do not fully understand why loss of a ubiquitously expressed protein leads to the distinct SMA pathogenesis. While we know that SMN depletion impacts first and foremost spinal cord motor neurons and skeletal muscle, it is now appreciated that major regulatory tissues such as the brain, liver and pancreas are also afflicted, causing specific debilitating symptoms [19]. Understanding the developmental and tissue requirements of SMN will be of utmost importance for refining the timing and delivery methods of $S M N$ gene therapies, thus ultimately optimizing their efficiencies.

Furthermore, a large number of current research endeavours are aimed at identifying non-SMN pathways that are aberrantly regulated in SMA that contribute to disease progression [11]. Novel therapeutic entities such as commercially available drugs and dietary interventions that target these pathways can then be evaluated in wellcharacterized cellular and animal models, where their impact on functional disease phenotypes can be assessed [7,11]. These non-SMN treatments are not aimed at replacing $S M N$ gene therapies, but instead, at being delivered alongside them, in the form of a combinatorial approach to treating SMA [7,11]. Realistically, it is not until 20-50 years from now that we will really appreciate the impact of $S M N$ gene therapies on SMA patients, at which point we will have a greater awareness of the clinical symptoms that they may still present and the associated therapeutic needs [8]. It is therefore particularly crucial that we be one step ahead, anticipating what these treatment requirements will be.

It is fair to say that others in the fields of neuromuscular, neurodegenerative and monogenic research are looking at the SMA community as leaders and trailblazers in the successful development of an efficient gene therapy from bench to bedside. It therefore becomes essential that we continue leading the way by addressing all of the remaining questions discussed above as well as anticipating new ones, thus ensuring that the $S M N$ gene therapies that have been developed are able to provide maximal health benefits across the life course of all SMA patients. This can only occur with a steady flow of funding from both charities and federal/government funding bodies [20]. Thus, while we have changed the course of the disease for the better, it is important that we continue funding fundamental, pre-clinical and clinical SMA research, to keep us on an upward trajectory, in the hopes that one day, no one has to succumb to SMA.

Financial \& competing interests disclosure

The authors are grateful to SMA Angels Charity, Muscular Dystrophy UK, Association Française contre les Myopathies, Action Medical Research for Children and SMA Support UK for funding our research on the development of combinatorial therapies for spinal muscular atrophy. The authors have no other relevant affiliations or financial involvement with any organization or entity with a financial interest in or financial conflict with the subject matter or materials discussed in the manuscript apart from those disclosed.

No writing assistance was utilized in the production of this manuscript. 


\section{Open access}

This work is licensed under the Creative Commons Attribution-NonCommercial-NoDerivatives 4.0 Unported License. To view a copy of this license, visit: http://creativecommons.org/licenses/by-nc-nd/4.0/

\section{References}

1. Pearn J. Incidence, prevalence, and gene frequency studies of chronic childhood spinal muscular atrophy. J. Med. Genet. 15(6), 409-413 (1978).

2. Crawford TO, Pardo CA. The neurobiology of childhood spinal muscular atrophy. Neurobiol. Dis. 3(2), 97-110 (1996).

3. Lefebvre S, Bürglen L, Reboullet $S$ et al. Identification and characterization of a spinal muscular atrophy-determining gene. Cell. 80(1), 155-165 (1995).

4. Lorson CL, Hahnen E, Androphy EJ, Wirth B. A single nucleotide in the SMN gene regulates splicing and is responsible for spinal muscular atrophy. Proc. Natl Acad. Sci. USA 96(11), 6307-6311 (1999).

5. Schrank B, Götz R, Gunnersen JM et al. Inactivation of the survival motor neuron gene, a candidate gene for human spinal muscular atrophy, leads to massive cell death in early mouse embryos. Proc. Natl Acad. Sci. USA 94(18), 9920-9925 (1997).

6. Lefebvre S, Burlet P, Liu Q et al. Correlation between severity and SMN protein level in spinal muscular atrophy. Nat. Genet. 16(3), 265-269 (1997).

7. Wood MJA, Talbot K, Bowerman M. Spinal muscular atrophy: antisense oligonucleotide therapy opens the door to an integrated therapeutic landscape. Hum. Mol. Genet. 26(R2), R151-R159 (2017).

8. Gidaro T, Servais L. Nusinersen treatment of spinal muscular atrophy: current knowledge and existing gaps. Dev. Med. Child Neurol. 61(1), 19-24 (2019).

9. Finkel RS, Mercuri E, Darras BT et al. Nusinersen versus sham control in infantile-onset spinal muscular atrophy. N. Engl. J. Med. 377(18), 1723-1732 (2017).

10. Finkel RS, Chiriboga CA, Vajsar J et al. Treatment of infantile-onset spinal muscular atrophy with nusinersen: a phase 2, open-label, dose-escalation study. Lancet Lond. Engl. 388(10063), 3017-3026 (2016).

11. Bowerman M, Becker CG, Yáńez-Muñoz RJ et al. Therapeutic strategies for spinal muscular atrophy: SMN and beyond. Dis. Model. Mech. 10(8), 943-954 (2017).

12. Pechmann A, Langer T, Schorling D et al. Evaluation of children with SMA type 1 under treatment with Nusinersen within the expanded access program in germany. J. Neuromuscul. Dis. 5(2), 135-143 (2018).

13. Messina S, Pane M, Sansone V et al. Expanded access program with Nusinersen in SMA type I in Italy: strengths and pitfalls of a successful experience. Neuromuscul. Disord. 27(12), 1084-1086 (2017).

14. Aragon-Gawinska K, Seferian AM, Daron A et al. Nusinersen in patients older than 7 months with spinal muscular atrophy type 1: a cohort study. Neurology 91(14), e1312-e1318 (2018).

15. Farrar MA, Teoh HL, Carey KA et al. Nusinersen for SMA: expanded access programme. J. Neurol. Neurosurg. Psychiatry. 89(9), 937-942 (2018).

16. Bertini E, Hwu W-L, Reyna SP et al. Efficacy and safety of nusinersen in infants with presymptomatic spinal muscular atrophy (SMA): Interim results from the NURTURE study. Eur. J. Paediatr. Neurol. 21, e14 (2017).

17. Montes J, Young SD, Mazzone E et al. Ambulatory function and fatigue in nusinersen-treated children with spinal muscular atrophy. (P2.322). Neurology 90(15 Supplement), P2.322 (2018).

18. Pacione M, Siskind CE, Day JW, Tabor HK. Perspectives on Spinraza (Nusinersen) treatment study: views of individuals and parents of children diagnosed with spinal muscular atrophy. J. Neuromuscul. Dis. 6(1), 119-131 (2018).

19. Hamilton G, Gillingwater TH. Spinal muscular atrophy: going beyond the motor neuron. Trends Mol. Med. 19(1), 40-50 (2013).

20. Christie-Brown V, Mitchell J, Talbot K. The SMA Trust: the role of a disease-focused research charity in developing treatments for SMA. Gene Ther. 24(9), 544-546 (2017). 
